\title{
FINITE ELEMENT FREE SURFACE SEEPAGE ANALYSIS WITHOUT MESH ITERATION
}

\author{
KLAUS-JÜRGEN BATHE* AND MOHAMMAD R. KHOSHGOFTAAR \\ Department of Mechanical Engineering, Massachusetts Institute of Technology, Cambridge, Massachusetts 02139, \\ U.S.A.
}

\section{SUMMARY}

An effective solution procedure for the finite element analysis of free surface seepage problems is presented. The solution algorithm employs a non-linear permeability description of the material and avoids iteration with the finite element mesh. The results and experiences obtained in the analyses of some problems are presented to demonstrate the usefulness of the technique.

\section{INTRODUCTION}

The phenomena of fluid flow or seepage through porous media is observed in various disciplines of engineering. ${ }^{1,2}$ It appears therefore natural that, as soon as the generality of the finite element method of analysis was recognized, emphasis was directed to develop the finite element method also for analysis of seepage problems in order to obtain a more general analysis tool. ${ }^{3}$ Apart from being able to consider in an effective manner complex geometries and material properties, emphasis on the development of the finite element analysis procedures is also important because of the potential of the technique for analysis of coupled stress and fluid flow problems. 4,5

The current practice using the finite element method in the analysis of free surface fluid flow through porous media is to assume a free surface, discretize the domain below the free surface using finite elements, solve for the flow conditions in the finite element model, and check whether the free surface boundary conditions are satisfied with sufficient accuracy. If the flow conditions at the free surface are not satisfied to a specified tolerance, the free surface is adjusted and the problem is resolved until the free surface flow conditions are met. Depending on the problems considered, some 10 to 30 iterations may be necessary in steady-state analysis, and in transient analysis an iteration is carried out in the time steps of the time response calculation.

In the iteration for the free surface, each iteration step represents a new problem, and a new finite element mesh could be established in each step. However, to keep the analysis effort to a minimum, usually the same basic finite element mesh is employed, but the geometric locations of the nodal points (possibly only near the free surface) are adjusted. The disadvantages of this scheme are that the elements can become very distorted, thus introducing severe errors in the analysis, and that a relatively large computational effort is required. These disadvantages are particularly pronounced in three-dimensional analysis. If non-linear stress and flow conditions

\footnotetext{
* Associate Professor.

$\ddagger$ Research Assistant. 
are analysed, the change in the geometric locations of the elements introduces additional difficulties that arise because the internal variables of the analysis are monitored at the integration points of the elements which change location. To overcome these difficulties Desai recently proposed a scheme for analysis of unconfined seepage, in which the original finite element mesh is not changed and the free surface is located using a relaxation type iterative scheme. $^{6}$

The objective in this paper is to present a very simple but effective procedure for analysis of unconfined seepage conditions. In the solution the original finite element mesh is not changed, and the free surface conditions are incorporated by use of a non-linear permeability behaviour of the solid material. Various sample solutions of unconfined seepage are presented and discussed using the solution scheme. In this paper only steady-state conditions are considered and the seepage analysis is carried out decoupled from the stress analysis; however, the proposed technique is also applicable to the solution of other free surface flow problems.

\section{GOVERNING EQUATIONS OF SEEPAGE PROBLEMS}

We consider the steady-state saturated flow conditions through a dam as shown for a twodimensional flow condition in Figure 1. The differential equation governing the flow in the porous medium is

$$
\frac{\partial}{\partial x}\left(k_{x} \frac{\partial \phi}{\partial x}\right)+\frac{\partial}{\partial y}\left(k_{y} \frac{\partial \phi}{\partial y}\right)+\frac{\partial}{\partial z}\left(k_{z} \frac{\partial \phi}{\partial z}\right)=0
$$

where $k_{x}, k_{y}, k_{z}$ are the coefficients of permeability in the $x, y$, and $z$ principal directions of permeability, respectively, and $\phi$ is the fluid total head or potential

$$
\phi=z+\frac{p}{\gamma}
$$

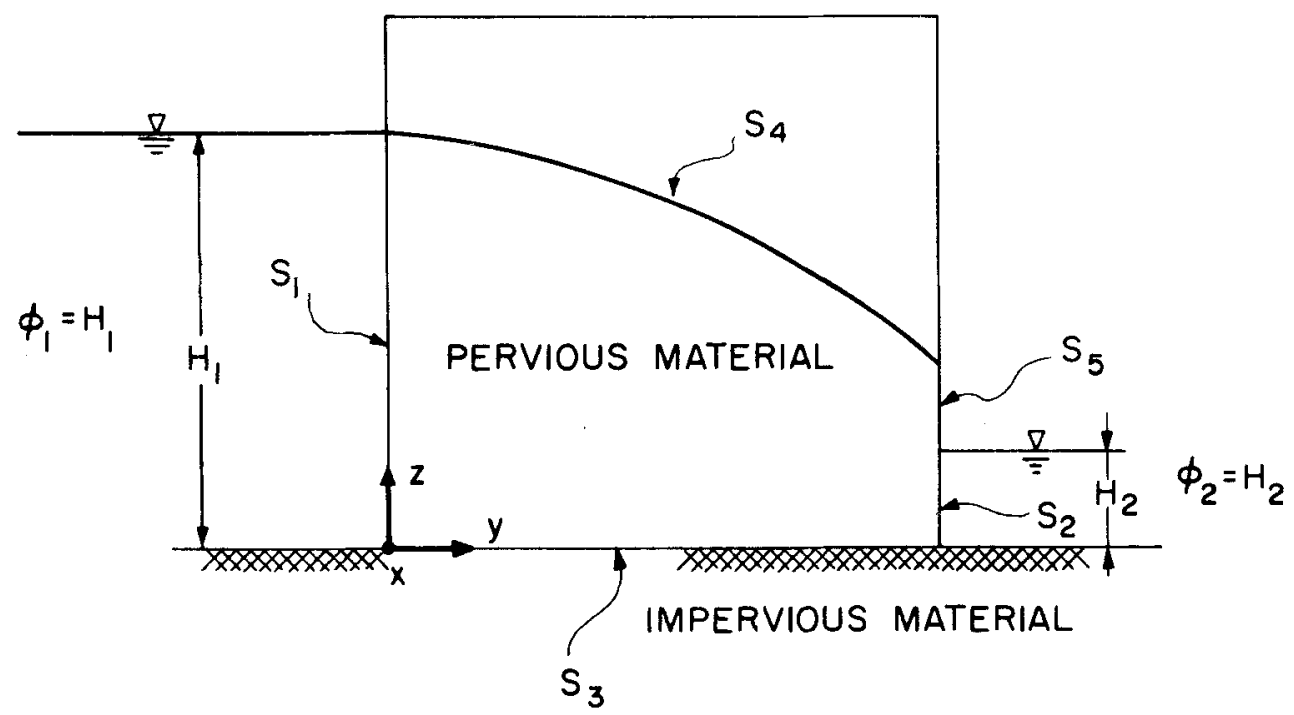

Figure 1. Steady unconfined flow through a dam 
In equation (2) $p$ is the fluid pressure, $\gamma$ is the unit weight of fluid and $z$ is the elevation at the point under consideration. The boundary conditions are:

for the upstream and downstream faces

$$
\begin{aligned}
& \phi=\phi_{1} \text { on } S_{1} \\
& \phi=\phi_{2} \text { on } S_{2}
\end{aligned}
$$

for the impervious base

$$
\frac{\partial \phi}{\partial n}=0 \text { on } S_{3}
$$

where $n$ denotes the normal to the surface;

for the free surface

$$
\left.\begin{array}{r}
\phi=z \\
\frac{\partial \phi}{\partial n}=0
\end{array}\right\} \text { on } S_{4}
$$

for the surface of seepage

$$
\phi=z \text { on } S_{5}
$$

In the finite element analysis a variational statement of the problem is employed in which the essential boundary conditions, expressed in equations (3), (4), (6) and (8), are explicitly imposed onto the solution, and the natural boundary conditions given in equations (5) and (7) are satisfied in an integrated sense by specifying zero flow input on $S_{3}$ and $S_{4}$. The virtual-work principle governing the steady state seepage flow is

$$
\int_{V} \delta \phi^{\prime \mathbf{T}} \mathbf{k} \phi^{\prime} \mathrm{d} V=0
$$

where $\delta$ denotes 'variation in', $\mathbf{k}$ is the permeability matrix in the principal material directions, i.e.,

$$
\mathbf{k}=\left[\begin{array}{ccc}
k_{x} & 0 & 0 \\
0 & k_{y} & 0 \\
0 & 0 & k_{z}
\end{array}\right]
$$

and

$$
\boldsymbol{\phi}^{\prime}=\left[\begin{array}{l}
\frac{\partial \phi}{\partial x} \\
\frac{\partial \phi}{\partial y} \\
\frac{\partial \phi}{\partial z}
\end{array}\right]
$$

For the finite element solution it is convenient to consider in equation (9) the complete domain of seepage including the boundaries and impose the essential boundary conditions (prescribed total potentials) at a later stage of solution. 


\section{FINITE ELEMENT FORMULATION}

An effective finite element solution is obtained using variable-number-nodes isoparametric elements, in which for an element with $N$ nodes $^{7}$

$$
\begin{gathered}
x=\sum_{i=1}^{N} h_{i} x_{i}, \quad y=\sum_{i=1}^{N} h_{i} y_{i} \\
z=\sum_{i=1}^{N} h_{i} z_{i}
\end{gathered}
$$

and

$$
\phi=\sum_{i=1}^{N} h_{i} \phi_{i}
$$

In equation (12) the $x_{i}, y_{i}$ and $z_{i}$ are the finite element nodal point coordinates, and the $h_{i}$ are the element interpolation functions. Similarly, in equation (13) the $\phi_{i}$ are the total potentials at the element nodes. Substituting the finite element assumption into equation (9) we obtain for the complete domain of seepage including the boundary surfaces ${ }^{7}$

$$
\mathbf{K}^{k} \boldsymbol{\phi}=\mathbf{0}
$$

where

$$
\mathbf{K}^{k}=\sum_{m} \int_{V^{(m)}} \mathbf{B}^{(m) \mathrm{T}} \mathbf{k}^{(m)} \mathbf{B}^{(m)} \mathrm{d} V^{(m)}
$$

with

$$
\begin{aligned}
& \mathbf{K}^{k}=\text { permeability matrix of total element assemblage } \\
& \phi=\text { vector of all nodal point total potentials } \\
& \mathbf{B}^{(m)}=\text { total potential gradient interpolation matrix of element } m \text { (Reference } 7, \text { p. 185) } \\
& \mathbf{k}^{(m)}=\text { permeability matrix of element } m \text { as defined in equation }(10)
\end{aligned}
$$

To impose now the total heads prescribed on the boundaries we add high permeability coefficients to the diagonal elements of $\mathbf{K}^{k}$ corresponding to the boundary nodes, and specify flow conditions that result into the given total potentials (Reference $7, p .187$ ). Thus equation (14) is modified to yield

$$
\left(\mathbf{K}^{k}+\mathbf{K}^{b}\right) \boldsymbol{\phi}=\mathbf{Q}^{b}
$$

where $\mathbf{K}^{b}$ is a diagonal matrix. The $i$ th diagonal element in $\mathbf{K}^{b}$ is equal to zero if $\phi_{i}$ is not prescribed and is otherwise equal to $k$, where $k \gg k_{i i}^{k}$. Correspondingly, the $i$ th entry in the vector $\mathbf{Q}^{b}$ is equal to zero if $\phi_{i}$ is not specified and is otherwise equal to $k \phi_{i}$. It may be noted that this procedure of specifying prescribed total potentials does not introduce any numerical difficulties in the solution of $\phi$ irrespective of the magnitude of $k$ employed, because $k$ is only added to the diagonal elements of $\mathbf{K}^{k}$.

Considering the natural boundary conditions, it should be noted that the conditions $\partial \phi / \partial n=$ 0 on $S_{3}$ and $S_{4}$ are imposed by not prescribing any flow normal to the surfaces in equation (9), and thus in equation (14).

The solution to the seepage problem could now be obtained if the free surface $S_{4}$ were known. The finite element discretization of the domain within the boundaries $S_{i}, i=1, \ldots, 5$ would be carried out, and the unknown nodal point total potentials could be solved using equation (16). However, with the location of $S_{4}$ unknown, it is usual practice to assume a free 
surface, solve equation (16) with not all boundary conditions imposed, check whether all boundary conditions are satisfied and iterate with the free surface $S_{4}$ (and thus with the finite element mesh) until a solution has been obtained which meets all boundary conditions.

The basic requirement in the above finite element mesh iteration solution is that there shall be no flow above the $\phi=z$ line. This requirement is satisfied by not representing the material above the free surface. The basis of the scheme presented here is that this requirement can be met more easily computationally by recasting the problem in a non-linear form, in which the natural boundary condition on the free surface in equation (7) is always satisfied (in an integrated sense) and iteration is performed to satisfy also the geometric boundary condition in equation (6). Assume that the complete dam is represented using a finite element discretization, and let the permeability of the elements be:

$$
\text { material permeability }=\left\{\begin{array}{l}
k \text { for } \phi \geqslant z \\
0 \text { for } \phi<z
\end{array}\right.
$$

then the elements above the free surface are effectively removed and those below the free surface are still active. The material permeability in equation (17) corresponds to a non-linear permeability as shown in Figure 2. The solution to the seepage problem can now be obtained

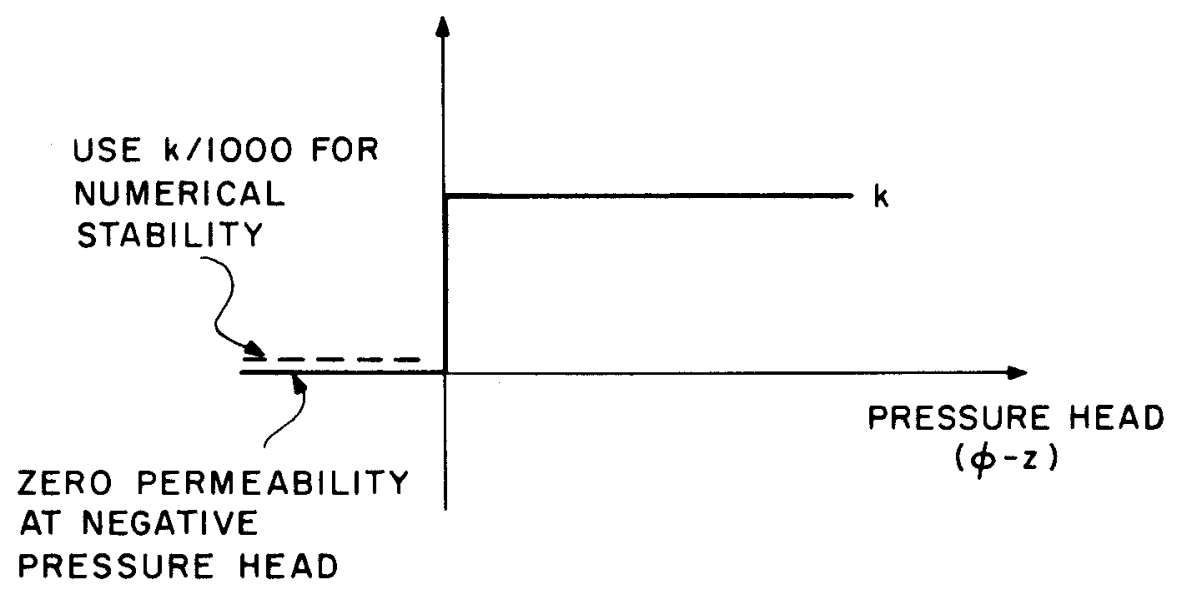

Figure 2. Material model employed for calculation of free surface

using a Newton-Raphson iteration. Namely, we recognize that instead of solving equation (16) we now want to operate on

$$
\mathbf{F}^{k}+\mathbf{F}^{b}-\mathbf{Q}^{b}=\mathbf{0}
$$

where

$$
\begin{gathered}
\mathbf{F}^{k}=\sum_{m} \int_{V^{(m)}} \mathbf{B}^{(m) \mathrm{T}}\left(\mathbf{k}^{(m)} \mathbf{B}^{(m)} \boldsymbol{\phi}\right) \mathrm{d} V^{(m)} \\
\mathbf{F}^{b}=\mathbf{K}^{b} \boldsymbol{\phi}
\end{gathered}
$$

and $\mathbf{k}^{(m)}$ is the non-linear pressure head dependent permeability matrix of element $m$. The elements of $\mathbf{k}^{(m)}$ are zero if $\phi<z$ and defined as in equation (10) if $\phi \geqslant z$. 
The Newton-Raphson iteration for solution of equation (18) is performed in the usual manner:

$$
\begin{gathered}
\left(\mathbf{K}^{k(i-1)}+\mathbf{K}^{b}\right) \Delta \boldsymbol{\phi}^{(i)}=\mathbf{Q}^{b}-\mathbf{F}^{k(i-1)}-\mathbf{F}^{b(i-1)} ; \quad i=1,2, \ldots \\
\mathbf{K}^{k(i-1)}=\sum_{m} \int_{V^{(m)}} \mathbf{B}^{(m) \mathrm{T}} \mathbf{k}^{(m)(i-1)} \mathbf{B}^{(m)} d V^{(m)} \\
\mathbf{F}^{k(i-1)}=\sum_{m} \int_{V^{(m)}} \mathbf{B}^{(m) \mathrm{T}}\left[\mathbf{k}^{(m)(i-1)} \mathbf{B}^{(m)} \boldsymbol{\phi}^{(i-1)}\right] \mathrm{d} V^{(m)} \\
\mathbf{F}^{b(i-1)}=\mathbf{K}^{b} \boldsymbol{\phi}^{(i-1)}
\end{gathered}
$$

and

$$
\phi^{(i)}=\phi^{(i-1)}+\Delta \phi^{(i)}
$$

In practice, it is frequently more effective to use the modified Newton iteration, in which case the permeability matrix is only established at the beginning of the iteration and is not updated. Thus, we use instead of equation (21),

$$
\left(\mathbf{K}^{k(0)}+\mathbf{K}^{b}\right) \Delta \boldsymbol{\phi}^{(i)}=\mathbf{Q}^{b}-\mathbf{F}^{k(i-1)}-\mathbf{F}^{b(i-1)}
$$

with convergence declared when $\left\|\Delta \boldsymbol{\phi}^{(i)}\right\|_{2} /\left\|\boldsymbol{\phi}^{(i)}\right\|_{2} \leqslant$ to $1^{7}$

\section{NUMERICAL AND CONVERGENCE CHARACTERISTICS}

The proposed scheme using a non-linear pressure dependent permeability for the solution is directly analogous to stress analysis, in which the stiffness of the material is set to zero once specific stress conditions are reached. ${ }^{8}$ Therefore, much of the experience available from these stress analyses is directly applicable to the seepage analysis.

For the seepage analysis it is recommended to employ in two-dimensional analysis 4- to 8-node isoparametric elements, and in three-dimensional analysis the corresponding 8- to 21 -node elements. ${ }^{7,8}$ Also, three- or four-point Gauss integration is in most cases effective. In order to preserve a positive definite coefficient matrix in equation (21) it is necessary to assign a small value of permeability to the material instead of an exact zero permeability (use about $k / 1000$ as shown in Figure 2).

As it is usual in non-linear isoparametric finite element analysis, in the numerical integration the appropriate material moduli are evaluated at the integration points, ${ }^{8}$ and therefore the free surface passes, in general, through the elements.

For solution the modified Newton iteration in equation (26) is probably in most analyses most effective. To specify initial conditions in the iteration, the total head at all nodal points can be set equal to the maximum total potential encountered in the problem, which renders the analysis procedure quite analogous to elastic-plastic stress analysis with an elastic stiffness matrix. Also, the non-zero elements in the matrix $\mathbf{K}^{b}$ of equation (21) need only correspond to the surfaces $S_{1}$ and $S_{2}$ in Figure 1 . Because of the material characteristics, convergence is assured, but a relatively large number of iterations may be required in some analyses. ${ }^{9}$ For the problems solved in this study, good convergence characteristics have been observed, and no convergence acceleration factors were needed. ${ }^{10}$

\section{SAMPLE SOLUTIONS}

To show the characteristics of the solution scheme we present in this section the analysis results and experiences obtained in the analyses of two dams. The solutions were obtained using the ADINAT computer program. ${ }^{11}$ 


\section{Unconfined flow through rectangular dam}

The steady-state free surface seepage through the rectangular dam shown in Figure 3 was analysed. The upstream and downstream water levels were maintained at sixteen and zero feet, respectively, and isotropic conditions with a constant permeability were assumed.

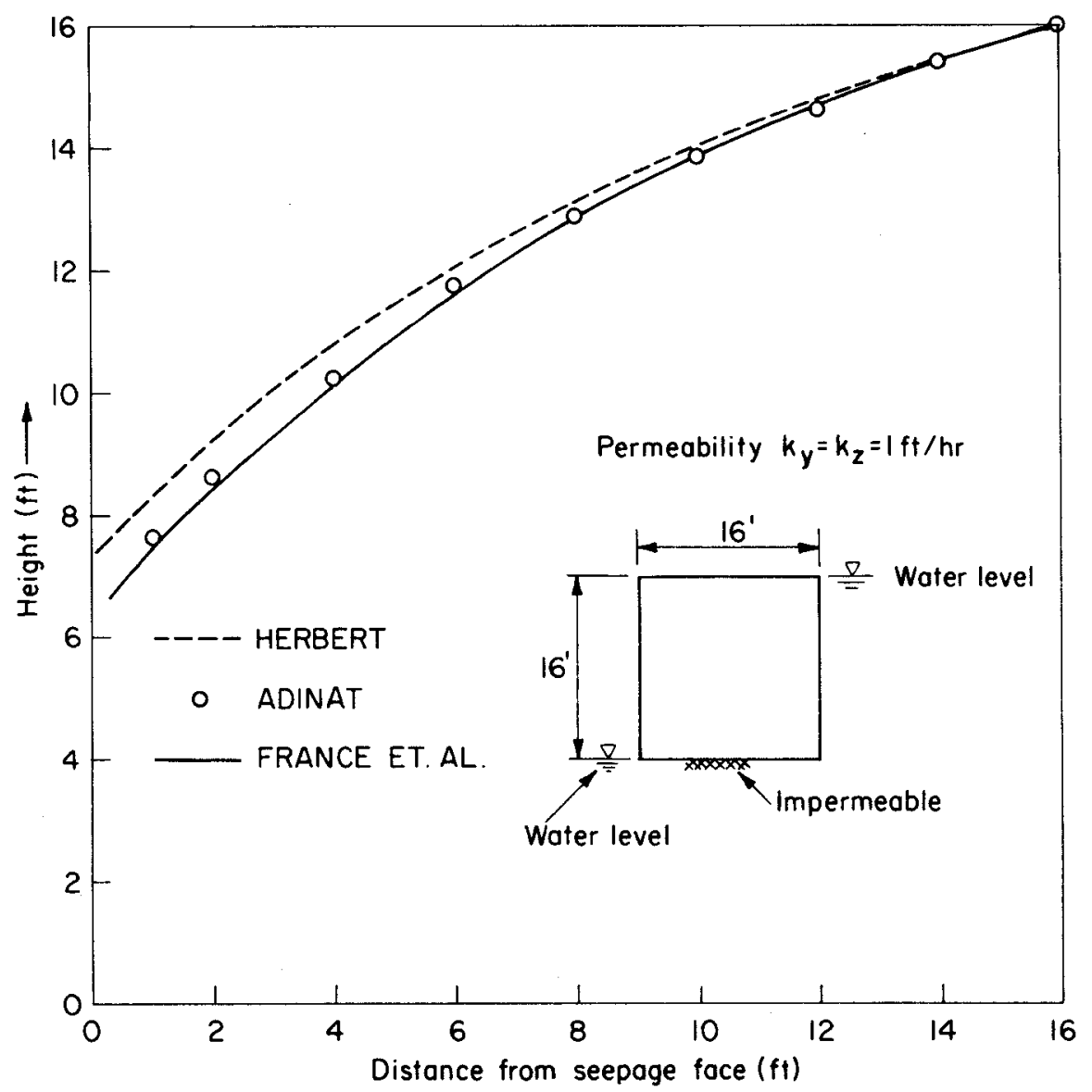

Figure 3. Steady-state unconfined flow through a porous block medium

The analysis was performed with two finite element models (see Figure 4). The first model consisted of $16 \times 16$ four-node equal elements, and the second model of $8 \times 8$ eight-node equal elements. Since the free surface flow line must in this problem lie above line AB shown in Figure 4, to reduce computations, the elements below that line were assumed to be linear, i.e., possess constant permeability properties in the iteration process. For the linear and non-linear elements two- and three-point Gauss numerical integration, respectively, was employed. The solution was obtained using the modified Newton iteration, in which the matrices $\mathbf{K}^{k}$ and $\mathbf{K}^{b}$ were calculated only once at the start of the solution. All elements in the starting iteration vector $\phi^{(0)}$ were taken to be equal to sixteen. 


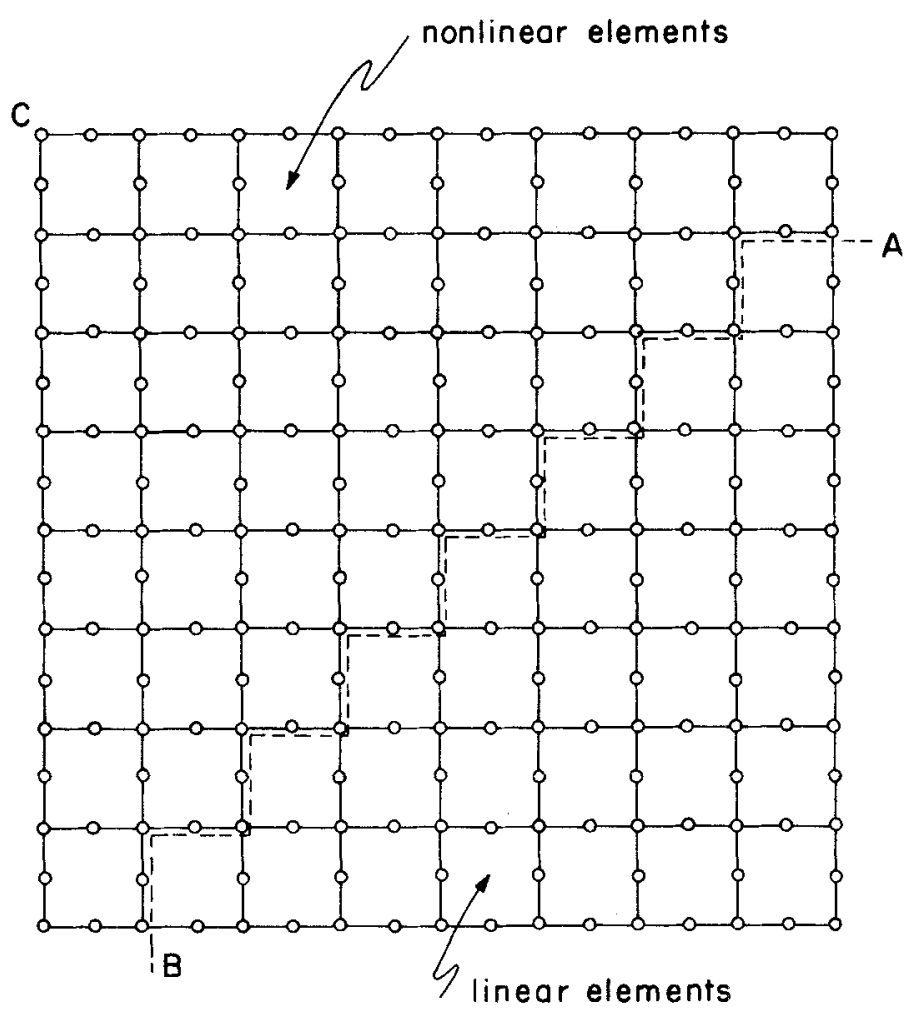

Figure 4. Finite element-mesh used for analysis of rectangular dam

Figure 3 shows the free surface calculated in this analysis and compares the analysis results with those of Herbert, ${ }^{12}$ who obtained an analogue solution, and France et al., ${ }^{13}$ who obtained a finite element solution. France et al. employed 20 iterations adjusting the finite element mesh to locate the free surface.

The four-node and eight-node element meshes used in this analysis gave the same results (to the degree of accuracy that can be drawn). Using the four-node element mesh, 6 iterations, and using the eight-node element mesh, 7 iterations were needed in the modified NewtonRaphson solution with tol $=0 \cdot 001$.

\section{Unconfined seepage through another dam}

The steady-state free surface seepage through the dam shown in Figure 5 was analysed. Figure 6 shows the finite element mesh employed. As in the analysis of the rectangular dam, in this analysis linear elements were used below the line AB (shown in Figure 6), two- and three-point Gauss integration was employed for the linear and non-linear elements, respectively, and the matrices $\mathbf{K}^{k}$ and $\mathbf{K}^{b}$ were calculated only once at the start of the modified Newton iteration. All elements in the starting iteration vector $\phi^{(0)}$ were taken to be equal to four.

Figure 5 shows the free surface calculated in this work and compares the results with those given by Harr, ${ }^{14}$ who obtained an analytical solution. In the modified Newton iteration 4 iterations were employed with tol $=0.001$. 


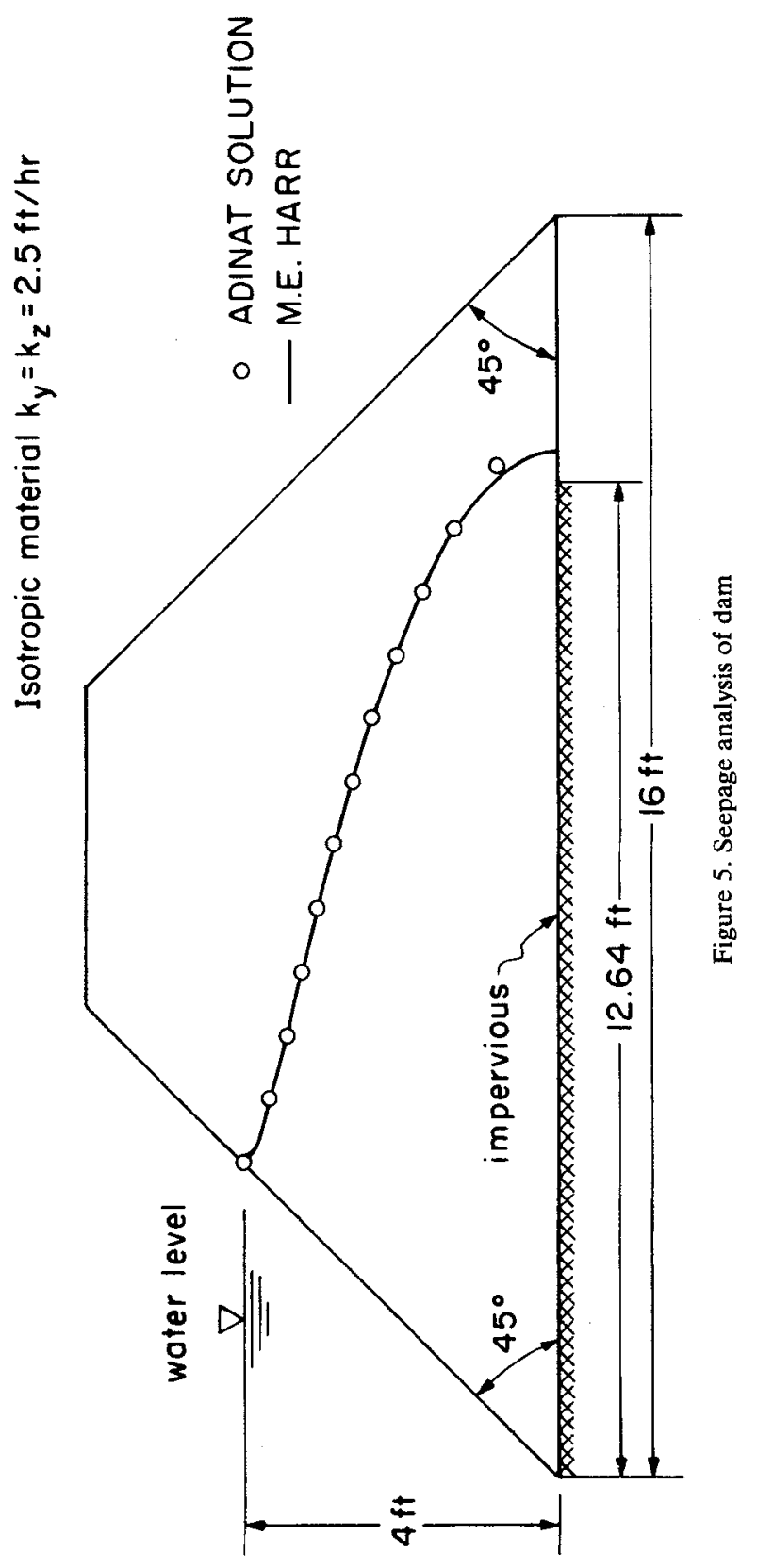




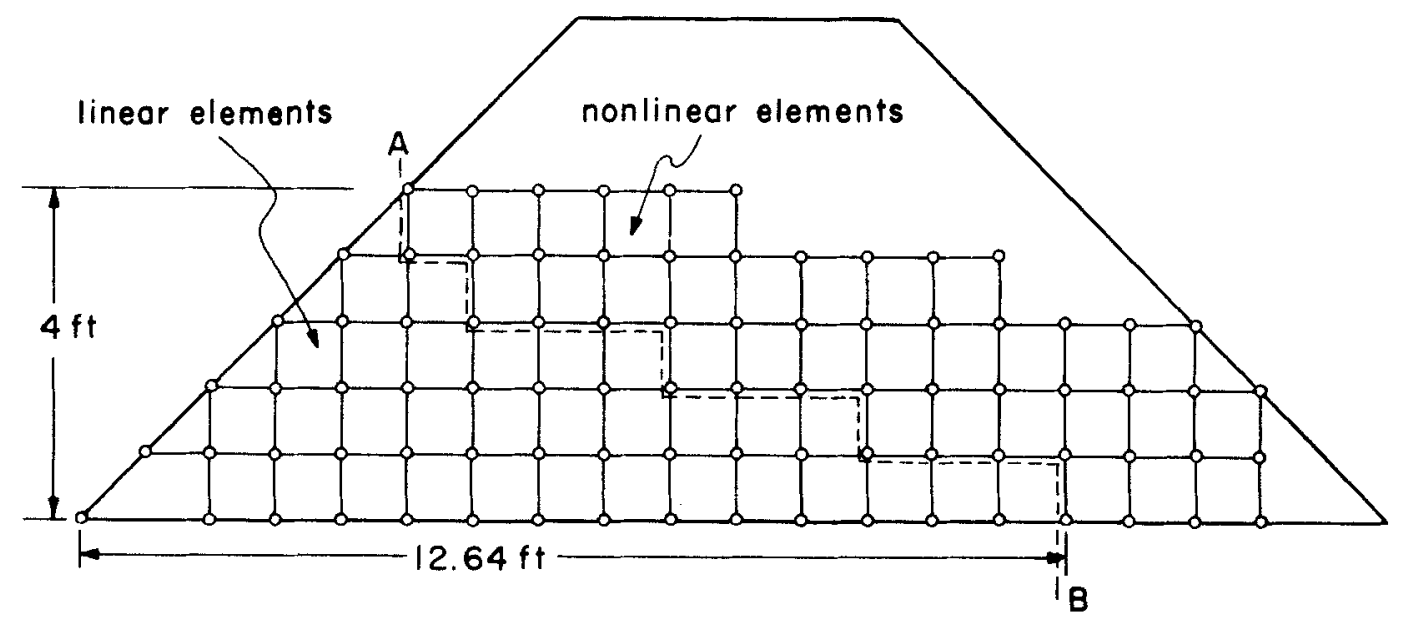

Figure 6. Finite element mesh used for analysis of dam

\section{CONCLUSIONS}

An effective solution procedure for the analysis of free surface seepage problems has been presented. The method does not require iteration with the finite element mesh, but instead uses a non-linear pressure dependent permeability description of the material and NewtonRaphson iteration. In the paper the application of the technique to the analysis of some steady-state flow conditions is given; however, the method should also be effective in transient seepage analysis, and in other free surface flow problems.

\section{ACKNOWLEDGEMENT}

The work reported in this paper has been financed by the ADINA users group. We would like to acknowledge gratefully this support.

\section{REFERENCES}

1. K. G. Stagg and O. C. Zienkiewicz, Rock Mechanics in Engineering Practice, Wiley, London, 1968.

2. A. Verruijt, Theory of Groundwater Flow, Gordon and Breach, New York, 1970.

3. C. S. Desai, 'Finite element methods for flow in porous media', Chap. 8 of Finite Elements in Fluids (Ed. R. H. Gallagher et al.), Wiley, London, 1975.

4. R. S. Sandhu and E. L. Wilson, 'Finite element analysis of seepage in elastic media', Proc. Am. Soc. Civ. Engng J. Engng Mech. Div., 95, 641-652 (1969).

5. J. Ghaboussi and E. L. Wilson, 'Flow of compressible fluid in porous elastic media', Int. J. num. Meth. Engng, 5, 419-442 (1973).

6. C. S. Desai, 'Finite element residual schemes for unconfined flow', Int. J. num. Meth. Engng, 10, 1415-1418 (1976).

7. K. J. Bathe and E. L. Wilson, Numerical Methods in Finite Element Analysis, Prentice-Hall Inc., Englewood Cliffs, N.J., 1976.

8. K. J. Bathe, 'Static and dynamic geometric and material nonlinear analysis using ADINA', AVL Report 82448-2, Mechanical Engineering Department, M.I.T. (May 1976) (revised May 1977).

9. K. J. Bathe, 'An assessment of current finite element analysis of nonlinear problems in solid mechanics', in Numerical Solution of Partial Differential Equations III SYNSPADE 1975 (Ed. B. Hubbard), Academic Press, New York, 1976.

10. G. C. Nayak and O. C. Zienkiewicz, 'Note on the 'Alpha' constant stiffness method for the analysis of non-linear problems', Int. J. num. Meth. Engng, 4, 579-582 (1972).

11. K. J. Bathe, 'ADINAT-A finite element program for automatic dynamic incremental nonlinear analysis of temperatures', AVL Report 82448-5, Mechanical Engineering Department, M.I.T. (May 1977).

12. R. Herbert, 'Time variant ground water flow by resistance network analogues', J. Hydrol., 6, 237-264 (1968).

13. P. W. France, C. J. Parekh, J. C. Peters and C. Taylor, 'Numerical analysis of free surface seepage problems', Proc. Am. Soc. Civ. Eng., J. Irigation and Drainage Div., 97, 165-179 (1971).

14. M. E. Harr, Groundwater and Seepage, McGraw-Hill, New York, 1962. 